\title{
Human Amniotic Membrane Promotes Antimicrobial Microenvironment in a Device-Related Infection
}

\author{
Alice S. Ferng1,2,3,4, Katherine M. Marsh ${ }^{1,2,5}$, Tia R. Pilikian', Alana Connell1,2, \\ Courtney Hemphill1,7, Samata Paidy ${ }^{5,8}$, Ray Runyan'3 , John Konhilas ${ }^{7}$, Zain Khalpey 1,2,3,4,7,8,9* \\ ${ }^{1}$ Department of Surgery, Division of Cardiothoracic Surgery, University of Arizona, Tucson, AZ, USA \\ ${ }^{2}$ College of Medicine-Tucson, University of Arizona, Tucson, AZ, USA \\ ${ }^{3}$ Department of Physiological Sciences, University of Arizona, Tucson, AZ, USA \\ ${ }^{4}$ Department of Biomedical Engineering, University of Arizona, Tucson, AZ, USA \\ ${ }^{5}$ Banner-University Medical Center, Tucson, AZ, USA \\ ${ }^{6}$ Amnio Technology LLC, Phoenix, AZ, USA \\ ${ }^{7}$ Department of Cellular and Molecular Medicine, University of Arizona, Tucson, AZ, USA \\ ${ }^{8}$ Department of Anesthesia, University of Arizona, Tucson, AZ, USA \\ ${ }^{9}$ Department of Medical Imaging, University of Arizona, Tucson, AZ, USA \\ Email: "Zkhalpey@surgery.arizona.edu
}

Received 15 December 2015; accepted 26 February 2016; published 29 February 2016

Copyright (C) 2016 by authors and Scientific Research Publishing Inc.

This work is licensed under the Creative Commons Attribution International License (CC BY).

http://creativecommons.org/licenses/by/4.0/

(c) () Open Access

\begin{abstract}
Hospital inquired post-operative infections are a leading cause of morbidity and mortality in patients receiving left ventricular assist devices. We present the case of a patient who acquired Candida albicans and Staphylococci infections following left ventricular assist device implantation. Use of standard antibiotics caused the infection to worsen, thus an amniotic membrane patch was used as a last-resort treatment. Within 6 weeks of the amniotic membrane patch treatment, the infection resolved, the wound appeared exceptionally clean upon inspection, and granulation tissue was present. After the infection resolved, a successful latissimusdorsi tissue flap procedure was completed and the patient made a full recovery.
\end{abstract}

\section{Keywords}

Antimicrobial, Amniotic Membrane, Ventricular Assist Device

\footnotetext{
${ }^{*}$ Corresponding author.
}

How to cite this paper: Ferng, A.S., Marsh, K.M., Pilikian, T.R., Connell, A., Hemphill, C., Paidy, S., Runyan, R., Konhilas, J. and Khalpey, Z. (2016) Human Amniotic Membrane Promotes Antimicrobial Microenvironment in a Device-Related Infection. J. Biomedical Science and Engineering, 9, 122-126. http://dx.doi.org/10.4236/jbise.2016.92008 


\section{Introduction}

Implantation of a left ventricular assist device (LVAD) is a well-established intervention as a bridge-to-transplantation or destination therapy in patients with heart failure. Infection is often a complication of LVAD placement and can lead to device malfunction, revision surgery, or device removal [1]. Additionally, patients with devices including LVADs may have iatrogenic immunologic dysfunction, thereby increasing the risk of postoperative infection [2] [3]. Infections resulting from LVAD implantation are most commonly treated by wound debridement, primary sternal closure, and mediastinal catheter irrigation with antibiotic or antiseptic solution [4]. Other treatment options include delayed closure with surgical reconstruction using vascularized tissue flaps from greater omentum or pectoral muscle and use of vacuum-assisted closure (VAC) [4]. Despite multiple treatment options for infection following cardiac surgery, evidence for optimal management of this postoperative complication is limited. Here, we present a patient who suffered persistent postoperative infections following robot-assisted Heart-Ware ${ }^{\circledR}$ left ventricular assist device (HVAD) implantation despite multiple sternal washouts and ideal antibiotic treatment. The anti-inflammatory properties of amniotic membrane patches have been previously demonstrated to be beneficial in cardiac surgery [5]. With this knowledge, our patient was given amniotic membrane patches as rescue therapy. This treatment resolved the infection and the patient was able to make a full recovery.

\section{Case History}

A 66 year-old male with a history of ischemic cardiomyopathy, chronic systolic heart failure, chronic coronary artery and kidney disease, and type 2 diabetes mellitus underwent robot-assisted HVAD implantation, left pericardial reconstruction using Goretex, and revision surgery two weeks after implantation. After device placement, purulent drainage was noted at the left apical incision scar. He returned to the operating room for a washout procedure with bacitracin, vancomycin, and Betadine; surgical culture results revealed 1+ Candida albicans and 2+ coagulase negative Staphylococci. He was intravenously administered metronidazole, moxifloxacin, vancomycin, and fluconazole in addition to undergoing two additional washouts without improvement. Due to his continued difficulties, the subsequent two irrigations included the same broad-spectrum antibiotics, but also included the placement of amniotic membrane. Cultures at the first amniotic membrane placement revealed residual 1+ Candida albicans plus newly developed 1+ Serratia. Following amniotic membrane placement, the wound was found to be approximately $80 \%$ free of purulent material. Given this marked improvement, the next washout also included amniotic membrane patch placement. Cultures revealed 2+ Candida albicans and 1+ anaerobic gram-positive Staphylococci. Resolution occurred within six weeks of the initial infection and the wound appeared clean upon inspection with good granulation tissue (Figure 1). After the infection resolved, a successful latissimus dorsitissue flap procedure was completed.

\section{Discussion}

Following heart failure, infection from device implantation is the second leading cause of mortality in LVAD patients [3]. Continuous antimicrobial therapy administered before, during and after implantation is most effective in reducing infection (Figure 2). However, patients receiving devices are at particularly high risk for the growth of antibiotic-resistant bacteria and other antibiotic-related complications when treated with continuous antimicrobial administration. Patients receiving devices have altered state of T-cell activation that promotes programmed apoptosis among CD4-bearing T cells [2]. Physical injury related to surgery increases the expression of Th2 lymphocytes that causes impaired cell mediated immunity. The Th1/Th2 ratio is then unbalanced, which can suppress the immune response. For example, decreased IL-12 expression and signaling results in a subsequent increase of $\mathrm{T}$ regulatory cells. Device patients therefore have immunologic dysfunction(s) and other risk factors including open or frequently treated wounds, thereby increasing their risk for infection.

Unique properties of the amniotic membrane patch may play a role in these pathways. Some of these properties include the facilitation of epithelial cell migration, reinforcement of basal cellular adhesion, and encouragement of cell differentiation [6]. This patch is isolated from placental tissue (hence it does not cause adverse immune responses) and consists of 3 main layers: the epithelial monolayer, a thick basement membrane, and avascular, hypocellular stromal matrix containing a non-viable heterogenous cell population (Figure 3). The membrane patch additionally contains numerous growth factors: epithelial growth factor (EGF), fibroblast growth factor (FGF), and TGF-beta 1, and others. EGF and FGF stimulate a similar, complement of intracellular signaling pathways. 


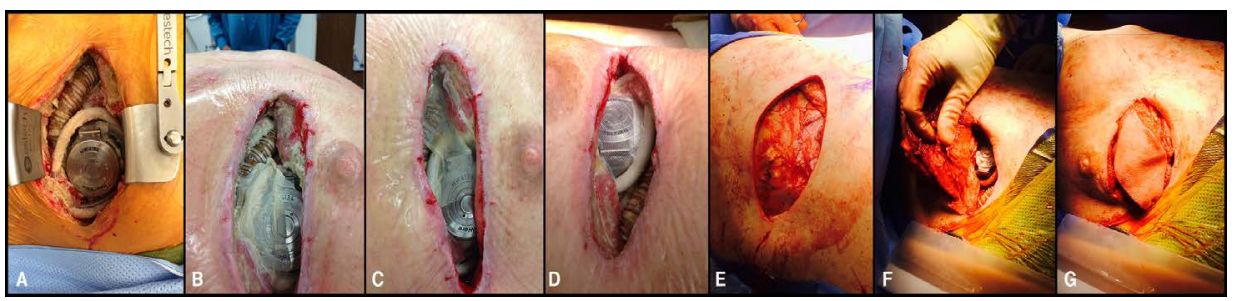

Figure 1. HVAD Device and Flap Placement. Initial robot-assisted HVAD implantation with left pericardial reconstruction using Goretex (A). Placement of an amniotic membrane patch at the device location 2.5 weeks after initial placement (B). Additional amniotic membrane patch placed 3 weeks after initial treatment (C). Device wound location 4 weeks after the initial placement (D). Following resolution of the infection after 2 amniotic membrane patch transplants over the course of 3 weeks, a latissimus dorsi tissue flap procedure (F), $(\mathrm{G})$ was successfully performed to repair and close the open wound $(\mathrm{E})$ at the HVAD implantation site at 4 weeks after the initial HVAD procedure.

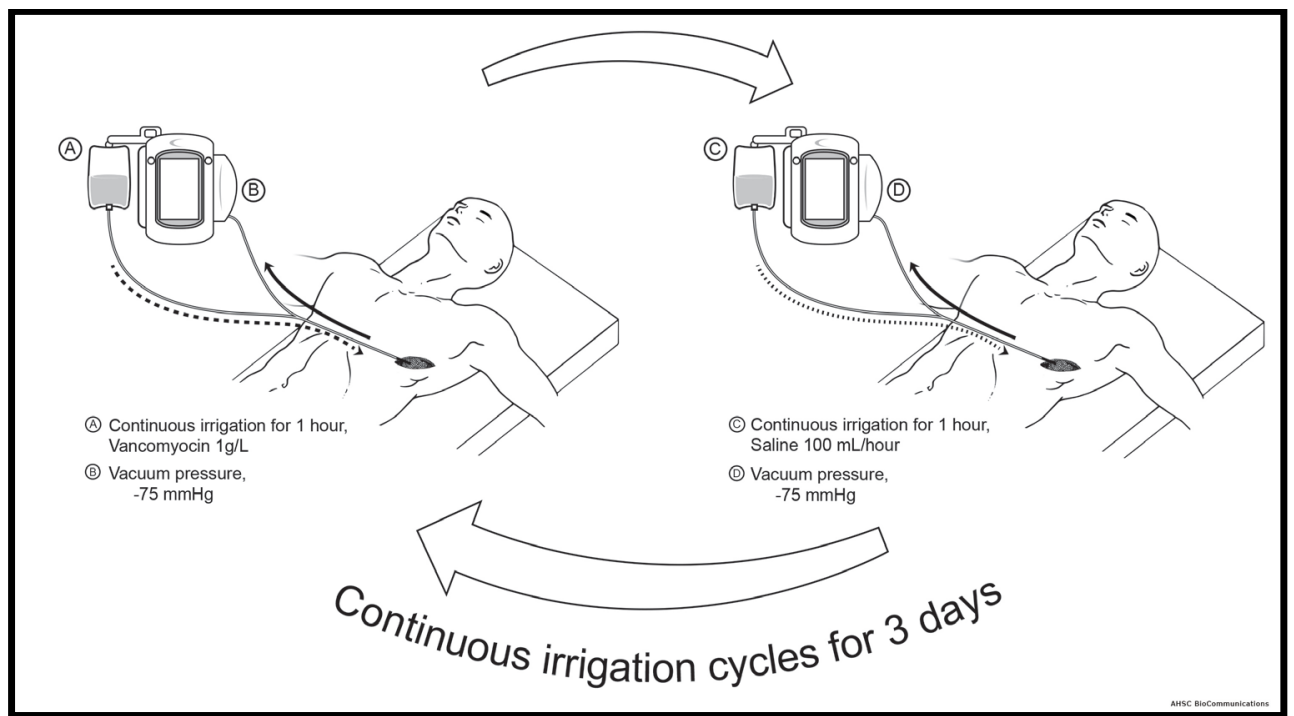

Figure 2. Washout of wound using continuous irrigation. The patient was treated with 2 washouts via continuous irrigation, performed sequentially from (A) to (D). Continuous irrigation at the wound site with antibiotics was performed for 1 hour (A), removed via vacuum (B), washed continuously with saline for 1 hour (C), and finally removed via vacuum (D).

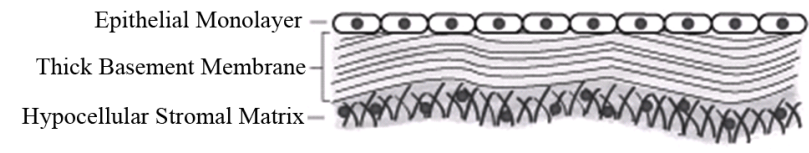

Figure 3. Amniotic Membrane Patch. The amniotic membrane patch is isolated from placental tissue and consists of 3 main layers: the epithelial monolayer, a thick basement membrane, and avascular, hypocellular stromal matrix containing a non-viable heterogenous cell population.

EGF aids in the recruitment of signaling proteins, and encourages cell growth, proliferation, and differentiation. FGF is largely involved in angiogenesis and wound healing, in addition to other endocrine signaling processes similar to EGF.

While TGF-beta 1 also possesses similar cell growth, proliferation, and differentiation properties, it additionally has a role in wound healing, especially in the heart. Since most immune cells secrete TGF-beta 1, this growth factor plays an important role in controlling the immune system. Some T cells release TGF-beta 1 to in- 
hibit the actions of other T cells. For example, TGF-beta 1 inhibits the action of IL-1, IL-2, helper T cells and cytotoxic T cells. It also down regulates the expression of certain cytokine receptors to help retard the inflammatory immune response. TGF-beta 1 similarly inhibits the action of other acute phase response cytokines including interferon-gamma and TNF-alpha. The amniotic membrane patch also contains antimicrobial peptides such as whey acidic peptide (WAP) and defensins [7]. All these elements together offer potential protection against bacterial, fungal and viral infections. For these reasons, the amniotic membrane patch has anti-inflammatory and antimicrobial properties which be used to modulate stromal scarring, and for external and internal wound healing applications.

\section{Conclusion}

The success of amniotic membrane patch placement in our device patient establishes this treatment as a potential intervention to decrease the incidence of postoperative infection. Given the minimal associated risks and positive preliminary outcomes, we suggest the amniotic membrane patch as a candidate for future research in this field.

\section{Acknowledgements}

The authors would like to acknowledge the patient and family.

\section{Disclosure}

The author (Zain Khalpey) of this publicationhas i) received research support from Amnio Technology, LLC, and ii) served as a paid consultant for Amnio Technology, LLC within the twenty-four month period preceding this publication. The terms of this arrangement have been reviewed and appropriately managed by the University of Arizona in accordance with its conflict of interest policies.

The author (Tia Pilikian) of the publication has received salary support from Amnio Technology LLC within the twenty-four month period preceding this publication.

\section{References}

[1] Aslam, S., Hernandez, M., Thornby, J., Zeluff, B. and Darouiche, R. (2010) Risk Factors and Outcomes of Fungal Ventricular-Assist Device Infections. Clinical Infectious Diseases, 50, 664-671. http://dx.doi.org/10.1086/650454

[2] Marik, P.E. and Flemmer, M. (2012) The Immune Response to Surgery and Trauma: Implications for Treatment. Journal of Trauma and Acute Care Surgery, 73, 801-808. http://dx.doi.org/10.1097/TA.0b013e318265cf87

[3] Califano, S., Pagani, F.D. and Malani, P.N. (2012) Left Ventricular Assist Device-Associated Infections. Infectious Disease Clinics of North America, 26, 77-87. http://dx.doi.org/10.1016/j.idc.2011.09.008

[4] Chan, M., Yusuf, E., Giulieri, S., Perrottet, N., Von Segesser, L., Borens, O. and Trampuz, A. (2015) A Retrospective Study of Deep Sternal Wound Infections: Clinical and Microbiological Characteristics, Treatment, and Risk Factors for Complications. Diagnostic Microbiology and Infectious Disease.

[5] Khalpey, Z., Marsh, K.M., Ferng, A., Riaz, I.B., Friedman, M., Indik, J., Avery, R., Jokerst, C. and Oliva, I. (2015) First in Man: Amniotic Patch Reduces Postoperative Inflammation. American Journal of Medicine, 128, e5-e6.

[6] Sangwan, V.S., Burman, S., Tejwani, S., Mahesh, S.P. and Murthy, R. (2007) Amniotic Membrane Transplantation: A Review of Current Indications in the Management of Ophthalmic Disorders. Indian Journal of Ophthalmology, 55, 251-260. http://dx.doi.org/10.4103/0301-4738.33036

[7] Ganz, T. (2003) Defensins: Antimicrobial Peptides of Innate Immunity. Nature Reviews Immunology, 3, 710-720. http://dx.doi.org/10.1038/nri1180 


\section{Abbreviations}

LVAD, left ventricular assist device

HVAD, heart ware ventricular assist device

EGF, epithelial growth factor

FGF, fibroblast growth factor

WAP, whey acidic peptide 